\title{
Studies on CGMS Based Short Duration Hybrids of Pigeonpea (Cajanus cajan (L.) Millsp.) in Terms of Combining Ability
}

\author{
J. Srivarsha ${ }^{1}$, J.E. Jahagirdar ${ }^{2}$, C.V. Sameer Kumar ${ }^{*}$, A.J. Hingane ${ }^{1}$, \\ D.K. Patil ${ }^{2}$, H.B. Shruthi ${ }^{1}$ and T.M. Bhosle ${ }^{3}$ \\ ${ }^{1}$ International Crops Research Institute for the Semi-Arid Tropics, Patancheru, \\ Telangana - 502324, India \\ ${ }^{2}$ Agriculture Research Station, Badnapur, Maharashtra, India \\ ${ }^{3}$ College of Agriculture, Badnapur, Vasantrao Naik Marathwada Krishi Vidyapeeth, \\ Parbhani, Maharashtra, India \\ *Corresponding author
}

A B S T R A C T

Keywords

CGMS, Combining ability, Randomized block design.

Article Info

Accepted:

26 September 2017

Available Online:

10 October 2017
To estimate combining ability, twenty seven hybrids were made from 12 parents in a line $\times$ tester mating design during Kharif 2015-16 and tested in a Randomized block design with three replications during Kharif 2016-17. Among these parents ICPL 161 and ICPL 149 had desirable GCA effect for grain yield per plant and its contributing characters. Ten crosses exhibited significant positive SCA effect for grain yield. Out of them most promising crosses in terms of grain yield were ICPA $2039 \times$ ICPL 161, ICPA $2156 \times$ ICPL 86022 and ICPA $2039 \times$ ICPL 90048. On the basis of per se performance and combining ability, the parents ICPA 2039, ICPL 88039, ICPL 161 and ICPL 149 can be used for future hybridization programmes.

\section{Introduction}

Pigeonpea [Cajanus cajan (L.) Millspaugh] is an important multipurpose grain legume crop primarily grown in tropical and subtropical areas of Asia, Africa and Latin America. The crop has multiple uses as food, fodder, fuel wood as well as for soil conservation and soil fertility enhancement. In particular, pigeonpea is both a crop and a food of the poor and plays an important role in food security and nutrition for some of the world's most disadvantaged people (Van der Maesen 1990). It also restores soil fertility by fixing atmospheric nitrogen and has the ability to solubilize fixed phosphorus (Ae et al.,1990). In India, pigeonpea is grown in an area of 5.21 million hectares with a production of 4.23 million tonnes (D. E. S, 2017).

Cytoplasmic-genic male sterility has been used since long time to improve the yield level of pigeonpea. This new hybrid pigeonpea technology is capable of substantially increasing the pigeonpea productivity (Saxena and Nadarajan, 2010). 
Identifying the parents with good combining ability can boost up the hybrid pigeonpea technology. Therefore, the present investigation was conducted to study the combining ability of three cytoplasmic male sterile lines with nine restorers to identify good general and specific combiners for grain yield and its components.

\section{Materials and Methods}

The materials under study comprise of three CMS lines (ICPA 2039, ICPA 2089 and ICPA 2156) and nine testers (ICPL 88034, ICPL 88039, ICPL 149, ICPL 161, ICPL 813, ICPL 89, ICPL 90048, ICPL 86022, ICPL 92047). These were crossed in an $\mathrm{L} \times \mathrm{T}$ fashion during the kharif 2015-16. The resultant $27 \mathrm{~F}_{1}$ 's along with 12 parents were planted in Randomized Block Design with three replications during kharif 2016-17. Each of the material under investigation was sown in four rows of four meters length with a spacing of $75 \times 25 \mathrm{~cm}$ between row to row and plant to plant respectively. Recommended and timely agronomic practices were taken up. Observations were recorded on five randomly selected plants for the traits viz.,Plant height, days to $50 \%$ flowering, pollen fertility, days to maturity, number of primary branches per plant, number of secondary branches per plant, number of pods per plant, number of seeds per pod, 100 seed weight, grain yield per plant, harvest index. The general combining ability (GCA) and specific combining ability (SCA) variances were worked out as per the method given by Kempthorne (1957).

\section{Results and Discussion}

In the Table 1, the analysis of variances of the study is presented. The genotypes were found highly significant for all the traits which indicated that the treatments used in this study were significantly different from each other.
The mean sum of squares (MSS) of the treatments was further partitioned into parent, cross and parents vs crosses. The results showed that all the parameters for parent, cross and parents vs crosses were found significant for plant height, days to $50 \%$ flowering, pollen fertility, days to maturity, number of secondary branches per plant, number of pods per plant, 100 seed weight, grain yield per plant and harvest index. The mean sum of squares in the analysis of variance due to lines were significant for all the characters except pollen fertility, number of primary branches per plant, number of seeds per pod and 100 seed weight indicating the importance of general combining ability and additive gene effects. The mean sum of squares due to testers were significant for the characters plant height and 100 seed weight indicating the importance of general combining ability and additive gene effects. The mean sum of squares due to line $x$ tester were significant for all the characters except number of primary branches per plant, number of secondary branches per plant and number of seeds per pod indicating the impact of specific combining ability and non-additive gene effects.

The proportional contribution of lines, testers, and line $\times$ testers for various characters are presented in Table 2 . The data revealed that contribution of line $\times$ tester was higher than both lines and testers for characters pollen fertility $(61.33 \%)$, days to maturity $(51.05 \%)$, number of primary branches per plant (45.64\%), number of secondary branches per plant $(45.64 \%)$, number of seeds per pod $(55.62 \%)$, grain yield per plant $(41.23 \%)$ and harvest index (65.98\%) indicating the preponderance of non-additive gene action for these characters. The contribution of tester was highest for plant height (41.13\%) and 100 seed weight $(51.94 \%)$ than line and line $\times$ tester. The contribution of lines was more than testers and line $\times$ tester for the character 
days to $50 \%$ flowering $(53.07 \%)$ and number of pods per plant $(43.97 \%)$ indicated that the concerned characters were influenced by additive gene action.

\section{Combining ability analysis}

Investigation of GCA effects (Table 3) revealed that the parents ICPA 2039 among lines, ICPL 161, ICPL 149, and ICPL 90048 among testers were the good general combiners for yield and most of the yield contributing characters. Hence these good general combiners of males and females may be extensively used in future for pigeonpea breeding programmes. The negative GCA effect was desirable in days to $50 \%$ flowering, days to maturity, which was observed in ICPA 2089, ICPA 2156 among lines and among testers it was observed in ICPL 88039. Among these parents, ICPL 161 and ICPL 149 had desirable GCA effect for grain yield per plant, plant height, number of secondary branches per plant, number of pods per plant, number of seeds per pod and harvest index. In general, good general combiners for grain yield also had good or average combining ability for one or more yield components. The GCA effects of lines and testers have been depicted in the graph 1 and 2. In most of the parents high GCA effects were associated with high per se mean for yield and yield components. It is important to mention here that the parents which showed good GCA effects for grain yield per plant also indicated significantly positive GCA effects for number of pods per plant. The results are in corroborance with the findings of Banu et al., (2006), Kumar et al., (2009), Vaghela et al., (2009), Shoba and Balan (2010) and Sudhir et al., (2017).

Specific combining ability effect is the index to determine usefulness of a particular combination in the exploitation of heterosis. The estimate of SCA effects of the hybrids are presented in Table 4. For the trait plant height, the cross ICPA $2089 \times$ ICPL 149 and ICPA $2039 \times$ ICPL 149 exhibited significant negative SCA effects. For days to $50 \%$ flowering and days to maturity negative SCA effects are desirable. Only one cross ICPA $2039 \times$ ICPL 88039 recorded significant negative SCA effect over both the traits. Only one cross recorded significant positive SCA effect for number of primary branches per plant viz., ICPA $2039 \times$ ICPL 81-3. For the trait number of secondary branches per plant, only one cross showed significant positive SCA effect viz., ICPA $2156 \times$ ICPL 88039.

Eight crosses exhibited significant positive SCA effect for pollen fertility. Maximum significant positive SCA effect was shown by ICPA $2156 \times$ ICPL 89 followed by ICPA $2156 \times$ ICPL 161. These results are in agreement with Wanjari et al., (2007).

For the trait number of pods per plant twelve crosses exhibited significant positive SCA effects. Maximum significant positive SCA effect was registered by ICPA $2039 \times$ ICPL 161 followed by ICPA $2156 \times$ ICPL 86022 . Only one cross recorded significant positive SCA effect viz., ICPA $2156 \times$ ICPL 90048 for the trait number of seeds per pod. For the trait 100 seed weight, three crosses exhibited significant positive SCA effects. Maximum significant positive SCA effect was registered by the cross ICPA $2089 \times$ ICPL 92047 .

Ten crosses exhibited significant positive SCA effect for grain yield. Most promising crosses in the order of their merit are ICPA $2039 \times$ ICPL 161 (35.63), ICPA $2156 \times$ ICPL 86022 (22.53), ICPA $2039 \times$ ICPL 90048 (24.02), ICPA $2089 \times$ ICPL 81-3 (15.01), ICPA $2089 \times$ ICPL 89 (11.56), ICPA $2039 \times$ ICPL 149 (8.85), ICPA $2156 \times$ ICPL 88039 (8.37), ICPA $2156 \times$ ICPL 89 (8.37) and ICPA $2156 \times$ ICPL 92047 (7.86) for grain yield per plant. 
Table.1 ANOVA for line $\times$ tester analysis

\begin{tabular}{|c|c|c|c|c|c|c|c|c|c|c|c|c|}
\hline \multirow[b]{2}{*}{$\begin{array}{c}\text { Sources of } \\
\text { variation }\end{array}$} & \multirow[b]{2}{*}{$\begin{array}{l}\text { d. } \\
\text { f. }\end{array}$} & \multicolumn{11}{|c|}{ Mean sum of squares } \\
\hline & & $\begin{array}{c}\text { Plant } \\
\text { height } \\
(\mathbf{c m})\end{array}$ & $\begin{array}{c}\text { Days to } 50 \\
\% \\
\text { flowering }\end{array}$ & $\begin{array}{l}\text { Pollen } \\
\text { fertility } \\
\quad(\%)\end{array}$ & $\begin{array}{l}\text { Days to } \\
\text { maturity }\end{array}$ & $\begin{array}{c}\text { No. of } \\
\text { primary } \\
\text { branches/ } \\
\text { plant }\end{array}$ & $\begin{array}{c}\text { No. of } \\
\text { secondary } \\
\text { branches } \\
\text { /plant }\end{array}$ & $\begin{array}{c}\text { No. of pods / } \\
\text { plant }\end{array}$ & $\begin{array}{c}\text { No. of } \\
\text { seeds / } \\
\text { pod }\end{array}$ & $\begin{array}{c}100 \\
\text { seed } \\
\text { weight } \\
(\mathrm{g})\end{array}$ & $\begin{array}{c}\text { Grain yield } \\
\text { / plant (g) }\end{array}$ & $\begin{array}{c}\text { Harvest } \\
\text { Index }(\%)\end{array}$ \\
\hline Treatment & 38 & $638.77^{* *}$ & $79.87^{* *}$ & $2046.23^{* *}$ & $126.53^{* *}$ & $1.58^{*}$ & $6.84^{* *}$ & $17430.13^{* *}$ & $0.08^{* *}$ & $0.94^{* *}$ & $1430.75^{* *}$ & $113.93^{* *}$ \\
\hline Replications & 2 & 123.96 & 14.80 & 1.70 & 40.26 & 2.05 & 2.78 & 384.34 & 0.04 & 0.06 & 11.36 & 0.89 \\
\hline Parent $(\mathrm{P})$ & 11 & $459.42^{* *}$ & $132.94^{* * *}$ & $6007.32^{* *}$ & $217.90^{* * *}$ & $3.30^{* * *}$ & $4.24^{*}$ & $18128.51^{* *}$ & $0.11^{* *}$ & $1.27^{* *}$ & $1307.45^{* *}$ & $113.83^{* *}$ \\
\hline Parents vs crosses & 1 & $5546.48^{* * *}$ & $87.48^{* *}$ & $9975.72^{* * *}$ & $835.56^{* *}$ & $3.22^{* * *}$ & $25.03^{* *}$ & $6935.13^{* *}$ & 0.01 & 0.61 & $507.67^{* *}$ & 30.23 \\
\hline Line & 2 & $2552.01^{* *}$ & $394.16^{* *}$ & 168.10 & $202.31^{*}$ & 1.27 & $25.76^{* *}$ & $100271.93^{* *}$ & 0.02 & 0.88 & $7695.10^{* *}$ & $189.37^{* *}$ \\
\hline Tester & 8 & $703.07^{*}$ & 45.15 & 40.18 & 45.85 & 1.07 & 7.91 & 13939.54 & 0.09 & $1.36^{*}$ & 975.95 & 82.22 \\
\hline Line xTester & 16 & $184.05^{* *}$ & $20.98^{* *}$ & $65.19^{* * *}$ & $50.28^{* * *}$ & 0.58 & 4.61 & $8996.00^{* *}$ & 0.06 & $0.52^{*}$ & $1017.57^{* * *}$ & $125.65^{* *}$ \\
\hline Error & 76 & 45.19 & 5.51 & 6.04 & 19.56 & 0.85 & 2.18 & 137.55 & 0.04 & 0.20 & 30.72 & 14.85 \\
\hline
\end{tabular}

- Significant at $5 \%$ level of significance, ${ }^{* *}$ - Significant at $1 \%$ level of significance

Note: A lines and B lines are isogenic except for pollen fertility. The observations of yield and yield contributing characters except pollen fertility were recorded on B-lines (ICPB 2039, ICPB 2089 and ICPB 2156).

Table. 2 Proportional contribution of lines, testers and line $\times$ tester

\begin{tabular}{|c|l|c|c|c|}
\hline Sr. No. & \multicolumn{1}{|c|}{ Characters } & Line (\%) & Tester (\%) & Line $\times$ tester (\%) \\
\hline 1. & Plant height (cm) & 37.32 & 41.13 & 21.53 \\
\hline 2. & Days to 50\% flowering & 53.07 & 24.32 & 22.06 \\
\hline 3. & Pollen fertility (\%) & 21.14 & 14.40 & 64.44 \\
\hline 4. & Days to maturity & 25.67 & 23.27 & 51.05 \\
\hline 5. & Number of primary branches / plant & 12.38 & 41.96 & 45.64 \\
\hline 6. & Number of secondary branches / plant & 27.33 & 33.55 & 39.11 \\
\hline 7. & Number of pods/ plant & 43.97 & 24.45 & 31.56 \\
\hline 8 & Number of seeds / pod & 2.21 & 42.16 & 55.62 \\
\hline 9. & 100 seed weight (g) & 8.42 & 51.94 & 39.63 \\
\hline 10. & Grain yield / plant (g) & 38.98 & 19.77 & 41.23 \\
\hline 11. & Harvest Index (\%) & 12.42 & 21.58 & 65.98 \\
\hline
\end{tabular}


Table.3 General combining ability of parents in pigeonpea

\begin{tabular}{|c|c|c|c|c|c|c|c|c|c|c|c|c|}
\hline $\begin{array}{l}\text { Sr. } \\
\text { No }\end{array}$ & Parents & $\begin{array}{c}\text { Plant } \\
\text { height } \\
\text { (cm) }\end{array}$ & $\begin{array}{c}\text { Days to } \\
50 \% \\
\text { flowering }\end{array}$ & $\begin{array}{c}\text { Pollen } \\
\text { fertility } \\
(\%)\end{array}$ & $\begin{array}{l}\text { Days to } \\
\text { maturity }\end{array}$ & $\begin{array}{c}\text { No. of } \\
\text { primary } \\
\text { branches } \\
\text { / plant }\end{array}$ & $\begin{array}{c}\text { No. of } \\
\text { secondary } \\
\text { branches } \\
\text { / plant }\end{array}$ & $\begin{array}{c}\text { No. of } \\
\text { pods per } \\
\text { plant }\end{array}$ & $\begin{array}{c}\text { No. of } \\
\text { seeds / } \\
\text { pod }\end{array}$ & $\begin{array}{c}100 \text { seed } \\
\text { wt. }(g)\end{array}$ & $\begin{array}{c}\text { Grain } \\
\text { yield } \\
\text { / plant (g) }\end{array}$ & $\begin{array}{c}\text { Harvest } \\
\text { Index } \\
(\%)\end{array}$ \\
\hline & Female parents & & & & & & & & & & & \\
\hline 1. & ICPA 2039 & $11.02^{* *}$ & $4.40^{* *}$ & $1.79^{* *}$ & $2.95^{* *}$ & 0.23 & $0.83^{* *}$ & $69.84^{* *}$ & -0.03 & -0.11 & $19.37^{* *}$ & $-2.90^{* *}$ \\
\hline 2. & ICPA 2089 & $-7.35^{* *}$ & $-2.53^{* *}$ & $-2.85^{* *}$ & $-2.46^{* *}$ & -0.20 & 0.25 & $-27.44^{* *}$ & 0.02 & -0.10 & $-7.78^{* *}$ & 0.61 \\
\hline \multirow[t]{2}{*}{3.} & ICPA 2156 & $-3.68^{* *}$ & $-1.86^{* *}$ & $1.06^{* *}$ & -0.49 & -0.04 & $-1.08^{* *}$ & $-42.40^{* *}$ & 0.01 & $0.21^{*}$ & $-11.59^{* *}$ & $2.29^{* *}$ \\
\hline & Male parents & & & & & & & & & & & \\
\hline 4. & ICPL 88034 & 0.52 & -0.68 & 0.86 & $-3.01^{*}$ & -0.20 & $1.15^{*}$ & $13.31^{* *}$ & -0.10 & -0.01 & 2.13 & $-5.70^{* *}$ \\
\hline 5. & ICPL 88039 & $-10.75^{* *}$ & $-2.46^{* *}$ & -1.22 & $-2.79^{*}$ & -0.34 & 0.03 & $-35.36^{* *}$ & $0.19^{*}$ & 0.14 & $-4.20^{*}$ & $2.85^{*}$ \\
\hline 6. & ICPL 149 & $15.63^{* *}$ & $2.99^{* *}$ & $2.48^{* *}$ & 2.21 & -0.24 & -1.05 & $50.95^{* *}$ & 0.03 & -0.31 & $9.68^{* *}$ & $2.37^{*}$ \\
\hline 7. & ICPL 161 & $4.97^{*}$ & $2.77^{* * *}$ & $-1.46^{*}$ & 2.65 & 0.09 & $1.11^{*}$ & $57.45^{* *}$ & -0.03 & $-0.53^{* * *}$ & $13.91^{\text {** }}$ & $2.68^{*}$ \\
\hline 8. & ICPL $81-3$ & $8.63^{* *}$ & $2.43^{* *}$ & -0.48 & 1.32 & 0.48 & 0.53 & -0.38 & -0.02 & 0.26 & 2.34 & 0.38 \\
\hline 9. & ICPL 89 & $-9.03^{* *}$ & -0.90 & $-3.34^{* *}$ & -0.57 & 0.34 & 0.49 & $-50.69^{* *}$ & -0.01 & 0.16 & $-14.20^{* *}$ & $-3.66^{* *}$ \\
\hline 10. & ICPL 90048 & $-5.75^{*}$ & -0.35 & 0.67 & 2.32 & 0.45 & -0.08 & -0.01 & $0.13^{*}$ & $0.77^{* *}$ & $8.85^{* *}$ & -1.56 \\
\hline 11. & ICPL 86022 & $-6.70^{* * *}$ & $3.12^{* *}$ & $3.50^{* *}$ & -2.12 & -0.29 & $-1.58^{* *}$ & $-48.26^{* *}$ & -0.09 & -0.17 & $-16.37^{* * *}$ & 1.60 \\
\hline \multirow[t]{3}{*}{12.} & ICPL 92047 & 2.48 & -0.68 & -1.00 & -0.01 & -0.31 & -0.59 & $12.98^{* * *}$ & -0.09 & $-0.32^{*}$ & -2.13 & 1.03 \\
\hline & $\mathrm{SE} \pm \mathrm{Gi}$ (line) & 1.29 & 0.49 & 0.009 & 0.82 & 0.17 & 0.31 & 2.30 & 0.04 & 0.09 & 1.04 & 0.68 \\
\hline & $\mathrm{SE} \pm \mathrm{Gj}$ (tester) & 2.23 & 0.85 & 0.016 & 1.42 & 0.30 & 0.54 & 3.98 & 0.07 & 0.16 & 1.81 & 1.18 \\
\hline
\end{tabular}

$*$ - Significant at $5 \%$ level of significance, ** - Significant at $1 \%$ level of significance

Note: A lines and B lines are isogenic except for pollen fertility. The observations of yield and yield contributing characters except pollen fertility were recorded on B-lines (ICPB 2039, ICPB 2089 and ICPB 2156). 
Table.4 Specific combining ability of crosses in pigeonpea

\begin{tabular}{|c|c|c|c|c|c|c|c|c|c|c|c|c|}
\hline $\begin{array}{l}\text { Sr. } \\
\text { No }\end{array}$ & Crosses & $\begin{array}{c}\text { Plant } \\
\text { height } \\
(\mathrm{cm})\end{array}$ & $\begin{array}{c}\text { Days to } \\
50 \% \\
\text { flowering }\end{array}$ & $\begin{array}{l}\text { Pollen } \\
\text { fertility } \\
(\%)\end{array}$ & \begin{tabular}{|c|} 
Days \\
to \\
maturity
\end{tabular} & $\begin{array}{c}\text { No. of } \\
\text { primary } \\
\text { branches } \\
\text { / nlant }\end{array}$ & $\begin{array}{c}\text { No. of } \\
\text { secondary } \\
\text { branches } \\
\text { / nlant } \\
\end{array}$ & $\begin{array}{l}\text { No. of } \\
\text { pods / } \\
\text { plant }\end{array}$ & $\begin{array}{l}\text { No. of } \\
\text { seeds } \\
\text { / pod }\end{array}$ & $\begin{array}{c}100 \\
\text { seed } \\
\text { wt. } \\
(\sigma)\end{array}$ & $\begin{array}{c}\text { Grain } \\
\text { yield } \\
\text { / Plant } \\
\text { (o) }\end{array}$ & $\begin{array}{c}\text { Harvest } \\
\text { Index } \\
(\%)\end{array}$ \\
\hline 1. & ICPA2039 × ICPL88034 & -6.69 & -2.28 & 0.06 & -4.51 & -0.18 & $-1.97^{*}$ & 12.25 & 0.14 & -0.01 & 3.74 & 1.10 \\
\hline 2. & ICPA2039 × ICPL88039 & -2.76 & $-4.84^{* *}$ & $4.09^{* *}$ & $-7.06^{* *}$ & -0.07 & 1.25 & $50.15^{* *}$ & -0.15 & -0.25 & $-15.92^{* *}$ & -0.55 \\
\hline 3. & ICPA2039 × ICPL149 & $8.20^{*}$ & 1.05 & -0.26 & 4.27 & -0.07 & -0.11 & 11.87 & -0.06 & $0.53^{*}$ & $8.85^{* *}$ & 1.07 \\
\hline 4. & ICPA2039 × ICPL161 & $7.86^{*}$ & 0.27 & -0.68 & 0.83 & -0.20 & -0.56 & $107.55^{* *}$ & 0.04 & $0.58^{*}$ & $35.63^{* *}$ & 0.09 \\
\hline 5. & ICPA2039 × ICPL81-3 & 2.53 & 1.94 & $3.14^{*}$ & 3.49 & $1.04^{*}$ & -0.58 & $-37.13^{* *}$ & -0.04 & 0.06 & $-12.47^{* *}$ & -1.60 \\
\hline 6. & ICPA2039 × ICPL89 & 0.86 & 0.94 & $-7.06^{* *}$ & 1.72 & -0.49 & 0.15 & $-49.18^{* *}$ & -0.01 & -0.51 & $-19.92^{* *}$ & -2.60 \\
\hline 7. & ICPA2039 × ICPL90048 & -6.56 & $3.38^{*}$ & -0.81 & 2.83 & 0.37 & 1.51 & $67.37^{* *}$ & -0.16 & 0.15 & $24.02^{* *}$ & 1.40 \\
\hline 8. & ICPA2039 × ICPL86022 & $-9.47^{*}$ & -1.17 & $-2.45^{*}$ & 0.27 & -0.30 & 0.83 & $-55.05^{* *}$ & 0.13 & -0.11 & $-19.59^{* *}$ & $7.22^{* *}$ \\
\hline 9. & ICPA2039 × ICPL92047 & 6.02 & 0.72 & $3.96^{* *}$ & -1.84 & -0.10 & -0.53 & -7.55 & 0.10 & -0.43 & -4.34 & $-6.13^{* *}$ \\
\hline 10. & ICPA2089 × ICPL88034 & -0.99 & -0.02 & $3.04^{*}$ & 1.23 & 0.07 & -0.59 & $17.66^{*}$ & -0.21 & 0.17 & 4.89 & 2.33 \\
\hline 11. & ICPA2089 × ICPL88039 & 2.15 & -0.91 & $4.23^{* *}$ & 0.35 & 0.09 & -0.41 & $25.80^{* *}$ & 0.14 & -0.17 & $7.56^{*}$ & 2.47 \\
\hline 12. & ICPA2089 × ICPL149 & $7.90^{*}$ & -0.69 & -0.08 & -4.65 & 0.24 & 0.94 & 2.48 & 0.16 & -0.43 & -3.33 & -3.74 \\
\hline 13. & ICPA2089 × ICPL161 & -5.43 & 0.53 & $-4.04^{* *}$ & 2.23 & -0.10 & 0.35 & $-43.23^{* *}$ & -0.01 & -0.34 & $-13.55^{* *}$ & -0.72 \\
\hline 14. & ICPA2089 × ICPL81-3 & -2.10 & -1.80 & $-4.62^{* *}$ & -1.10 & -0.49 & 0.45 & $52.35^{* *}$ & 0.02 & -0.26 & $15.01^{* *}$ & -0.51 \\
\hline 15. & ICPA2089 × ICPL89 & -1.10 & 1.20 & 0.01 & -0.54 & 0.34 & -0.01 & $25.10^{* *}$ & -0.03 & 0.04 & $11.56^{* *}$ & $12.08^{* *}$ \\
\hline 16. & ICPA2089 × ICPL90048 & 6.28 & -0.69 & 2.09 & 1.57 & -0.62 & 0.38 & $52.08^{* * *}$ & -0.11 & 0.06 & $-15.67^{* *}$ & $-11.26^{* *}$ \\
\hline 17. & ICPA2089 × ICPL86022 & 2.90 & 2.09 & $3.13^{*}$ & -2.65 & 0.29 & -0.66 & $-20.20^{* *}$ & -0.02 & 0.24 & -2.94 & -2.23 \\
\hline
\end{tabular}


Table.4 Continued......

\begin{tabular}{|c|c|c|c|c|c|c|c|c|c|c|c|c|}
\hline $\begin{array}{l}\text { Sr. } \\
\text { No }\end{array}$ & Crosses & $\begin{array}{c}\text { Plant } \\
\text { height } \\
(\mathrm{cm})\end{array}$ & $\begin{array}{c}\text { Days to } \\
50 \% \\
\text { flowering }\end{array}$ & $\begin{array}{l}\text { Pollen } \\
\text { fertility } \\
(\%)\end{array}$ & $\begin{array}{c}\text { Days } \\
\text { to } \\
\text { maturity }\end{array}$ & $\begin{array}{c}\text { No. of } \\
\text { primary } \\
\text { branches } \\
\text { / plant }\end{array}$ & $\begin{array}{c}\text { No. of } \\
\text { secondary } \\
\text { branches } \\
\text { / plant }\end{array}$ & $\begin{array}{l}\text { No. of } \\
\text { pods / } \\
\text { plant }\end{array}$ & $\begin{array}{c}\text { No. of } \\
\text { seeds } \\
\text { / pod }\end{array}$ & $\begin{array}{c}100 \\
\text { seed } \\
\text { wt. } \\
\text { (g) }\end{array}$ & $\begin{array}{c}\text { Grain } \\
\text { yield } \\
\text { / Plant } \\
\text { (g) }\end{array}$ & $\begin{array}{c}\text { Harvest } \\
\text { Index } \\
(\%)\end{array}$ \\
\hline 18. & ICPA2089 × ICPL92047 & $-9.61^{*}$ & 0.31 & $-3.76^{* * *}$ & 3.57 & 0.16 & -0.45 & -7.87 & 0.05 & $0.68^{*}$ & -3.52 & 1.57 \\
\hline 19. & ICPA2156 × ICPL88034 & $7.68^{*}$ & 2.31 & $-3.10^{*}$ & 3.27 & 0.11 & $2.57^{* *}$ & $-29.91^{* *}$ & 0.06 & -0.16 & $-8.63^{* *}$ & -3.44 \\
\hline 20. & ICPA2156 × ICPL88039 & 0.61 & $5.75^{* *}$ & $-8.32^{* *}$ & $6.72^{* *}$ & -0.02 & -0.84 & $24.35^{* *}$ & 0.01 & 0.42 & $8.37^{* *}$ & -1.92 \\
\hline 21. & ICPA2156 × ICPL149 & $-16.10^{* *}$ & -0.36 & 0.34 & 0.38 & -0.18 & -0.83 & $-14.36^{*}$ & -0.10 & -0.10 & -5.52 & 2.67 \\
\hline 22. & ICPA2156 × ICPL161 & -2.43 & -0.80 & $4.72^{* *}$ & -3.06 & 0.30 & 0.21 & $-64.32^{* *}$ & -0.04 & -0.24 & $-22.08^{* *}$ & 0.63 \\
\hline 23. & ICPA2156 × ICPL81-3 & -0.43 & -0.14 & 1.47 & -2.40 & -0.55 & 0.13 & $-15.23^{*}$ & 0.02 & 0.20 & -2.54 & 2.10 \\
\hline 24. & ICPA2156 × ICPL89 & 0.23 & -2.14 & $7.05^{* *}$ & -1.17 & 0.15 & -0.14 & $24.09^{*}$ & 0.04 & 0.47 & $8.37^{* *}$ & $-9.48^{* *}$ \\
\hline 25. & ICPA2156 × ICPL90048 & 0.28 & -2.69 & -1.28 & -4.40 & 0.24 & $-1.90^{*}$ & $-15.29^{*}$ & $0.26^{*}$ & -0.21 & $-8.35^{* *}$ & $9.86^{* *}$ \\
\hline 26. & ICPA2156 × ICPL86022 & 6.57 & -0.91 & -0.68 & 2.38 & 0.01 & -0.17 & $75.25^{* *}$ & -0.11 & -0.13 & $22.53^{* *}$ & $-4.99^{* *}$ \\
\hline \multirow[t]{3}{*}{27.} & ICPA2156 × ICPL92047 & 3.59 & -1.02 & -0.20 & -1.73 & -0.06 & 0.98 & $15.42^{*}$ & -0.15 & -0.25 & $7.86^{*}$ & $4.56^{*}$ \\
\hline & SEij & 3.87 & 1.47 & 0.12 & 2.46 & 0.53 & 0.95 & 6.90 & 0.13 & 0.27 & 3.14 & 2.05 \\
\hline & C.D. $5 \%$ & 7.78 & 2.72 & 2.37 & 5.12 & 1.07 & 1.71 & 13.59 & 0.23 & 0.52 & 6.42 & 4.46 \\
\hline
\end{tabular}

* - Significant at $5 \%$ level of significance, ** - Significant at $1 \%$ level of significance 
Graph.1 and 2 Graphs showing the GCA effects of lines and testers for the trait grain yield / plant
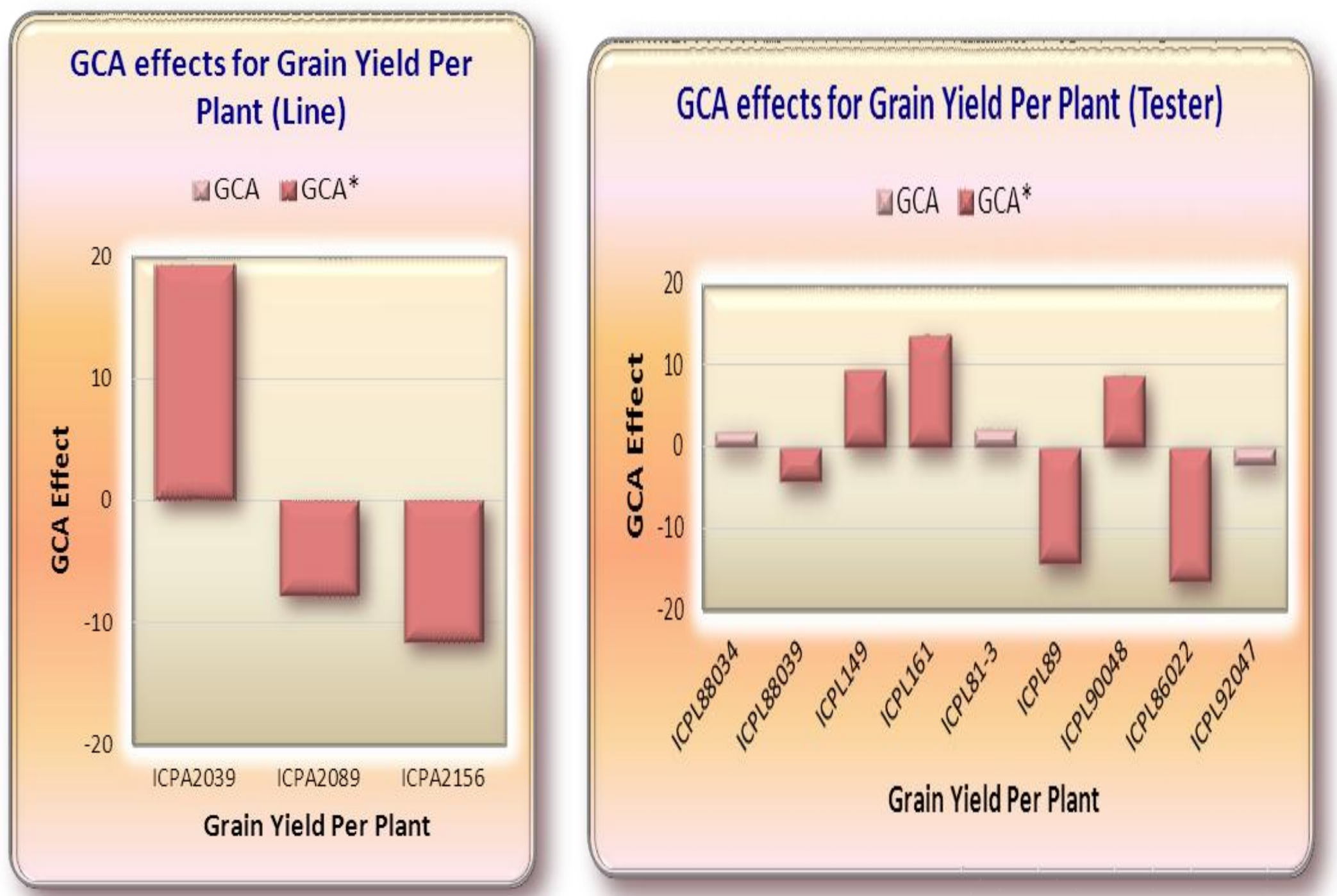
For the trait harvest index four crosses recorded significant positive SCA effects. Maximum significant positive SCA effect was exhibited by ICPA $2089 \times$ ICPL 89 followed by ICPA 2156 $\times$ ICPL 90048. These results are in agreement with the findings of Shoba and Balan (2010) and Gupta et al., (2011).

This study clearly indicated that there was no particular relationship between positive and significant SCA effects of crosses with GCA effects of their parents for all the characters under study. This was in agreement with the findings of Pandey et al., (2014) and Sudhir et al., (2017).

In crop improvement programme specific combining ability is important to pinpoint specific cross combination for commercial exploitation or varietal development. On the basis of per se performance and combining ability, the parents ICPA 2039, ICPL 88039, ICPL 161 and ICPL 149 can be used for future hybridization programmes. Promising crosses exhibiting significant positive SCA effect viz., ICPA $2039 \times$ ICPL 161, ICPA $2156 \times$ ICPL 86022 and ICPA $2039 \times$ ICPL 90048 for grain yield/plant may be considered for the hybrid breeding programme.

\section{References}

Ae, N., Arihara, J., Okada, K., Yoshihara, T and Johansen, C. 1990. Phosphorous uptake by pigeonpea and its role in cropping systems of the Indian subcontinent. Science. 248: 477-480.

Banu, M. R., Muthaiah, A. R. and Ashok, S. 2006. Combining ability studies in pigeonpea. Crop Research. 31(3):396-398.

D. E. S. 2017. Directorate of Economics and Statistics, Department of Agriculture and Cooperation. Based on second estimate of 2016-17.
Gupta, D. K., Acharya S. and Patel, J. B. 2011. Combining ability and heterosis studies in pigeonpea using $\mathrm{A}_{2}$ cytoplasm from Cajanus scarabaeoides as source of male sterility. J. Food Legumes. 24:58-64.

Kempthorne, O., 1957. An introduction to genetic statistics, John Wiley and Son Inc. Newyork, Champman and Hall Ltd. London, pp. 468-470.

Kumar, C. V. S., Sreelakshmi, C. H. and Varma, P. K. 2009. Studies on combining ability and heterosis in pigeon pea (Cajanus cajan L.). Legume Research. 32:92-97.

Pandey. P., Tiwari. D., Venkat.R. P., and Yadav. S. 2014. Studies on gene action and combining ability of cytoplasmic-genic male sterile hybrids in pigeonpea [Cajanus cajan (L.) Millsp.]. Australian Journal of Crop Science. 8(5): 814-821.

Saxena, K. B., and Nadarajan, N. 2010. Prospects of Pigeonpea hybrids in Indian Agriculture. Electronic Journal of Plant Breeding. 1:1107-1117.

Shoba, D., and Balan, A. 2010. Combining ability in CMS/GMS based pigeonpea [Cajanus cajan (L.) Millsp.] hybrids. Madras Agricultural Journal. 97:25-28.

Sudhir, K., Singh. P. K., Sameer Kumar, C. V. and Saxena, K. B. 2017. Study of CGMS based pigeonpea [Cajanus cajan (L.) Millsp] hybrids in terms of combining ability. Int. J. Agric. Sci. Res., 7(2): 129-134.

Vaghela, K. O., Desai, R. T., Nizama, J. R., Patel, J. D. and Sharma, V. 2009. Combining ability analysis in pigeonpea [Cajanus cajan (L.) Millsp.]. Legume Res., 32:274277.

Van der Maesen, L. G., J. 1990. Pigeonpea: origin, history, evolution and taxonomy. CAB International. Pp 44-87.

Wanjari, K. B., Bhongle, S. A. and Sable, N. H. 2007. Evaluation of heterosis in CMS based hybrids in pigeonpea. Journal of Food Legumes. 20(1):107-108.

\section{How to cite this article:}

Srivarsha, J., J.E. Jahagirdar, C.V. Sameer Kumar, A.J. Hingane, D.K. Patil, H.B. Shruthi and Bhosle, T.M. 2017. Studies on CGMS Based Short Duration Hybrids of Pigeonpea [Cajanus cajan (L.) Millsp.] in Terms of Combining Ability. Int.J.Curr.Microbiol.App.Sci. 6(10): 3128-3136. doi: https://doi.org/10.20546/ijcmas.2017.610.367 